\title{
Porque Não Deveríamos Esquecer o Preditor de Smith *
}

\author{
Gabriel F. Machado, René D. O. Pereira, \\ Bismark C. Torrico e Fabrício G. Nogueira \\ Departamento de Engenharia Elétrica, Universidade Federal do Ceará, \\ Fortaleza, Brasil \\ (e-mails: gabrielfreitas@alu.ufc.br,reneolimpio@alu.ufc.br, \\ bismark@dee.ufc.br,fnogueira@dee.ufc.br)
}

\begin{abstract}
Recently, the use of the Smith Predictor was questioned due to issues related to its robustness to process dead-time uncertainties. Thus, this work presents a comparison between a structure derived from Smith Predictor, called Simplified Dead-Time Compensator (SDTC), and $\mathrm{PI}$ PID controllers in terms of performance and robustness. The main results of this comparison show that the SDTC is better or similar than PI/PID controllers in terms of performance and that robustness to dead-time uncertainties can be better quantified in terms of multiplicative uncertainties and robustness index curves.

Resumo: Recentemente, o uso do Preditor de Smith foi questionado devido a problemas relacionadas à sua robustez à incertezas no atraso de transporte do processo. Assim, este trabalho apresenta uma comparação entre uma estrutura derivada do Preditor de Smith, denominada Compensador de Atraso de Transporte Simplificado (SDTC), e controladores PI/PID em termos de desempenho e robustez. Os principais resultados desta comparação mostram que o SDTC é melhor ou similar aos controladores PI/PID em desempenho e que a robustez quanto à incertezas no atraso de transporte pode ser melhor quantificada em termos de curvas de incerteza multiplicativa e de índice de robustez.
\end{abstract}

Keywords: Dead-Time; DTC; PI; PID; Performance-robustness trade-off; Delay margin. Palavras-chaves: Atraso de transporte; DTC; PI; PID; Compromisso desempenho-robustez; Margem de atraso.

\section{INTRODUÇÃO}

O Preditor de Smith (SP, do inglês Smith Predictor) foi o primeiro método de compensação de sistemas com atraso que obteve desempenho considerável frente aos controladores proporcional-integral (PI) e proporcional-integralderivativo (PID) convencionais para processos com longos atrasos (Smith, 1957). No entanto, sua aplicação se limita a processos estáveis em malha aberta. No caso de processos integradores, o SP não é capaz de rejeitar perturbações e no caso de processos instáveis em malha aberta, o sistema em malha fechada é instável para incertezas e perturbações infinitesimais. Como solução, ao longo dos anos foram propostas várias estruturas derivadas do SP que lidavam com estes tipos de processos, além de melhorar seu desempenho e robustez. Estes controladores são chamados de Compensadores de Atraso de Transporte (DTCs, do inglês Dead-Time Compensators). Uma ampla revisão de DTCs pode ser encontrada em Normey-Rico and Camacho (2007) e Visioli and Zhong (2011).

Uma das mais relevantes estruturas derivadas do SP, o Preditor de Smith Filtrado (FSP, do inglês Filtered Smith Predictor), foi proposta por Normey-Rico and Camacho

\footnotetext{
* As simulações completas deste artigo estão disponíveis na sua página no ResearchGate.
}

(2009). Ele é uma estrutura de dois graus de liberdade, com desacoplamento entre a resposta de seguimento de referência e a resposta de rejeição de perturbação. Além disso, o FSP é capaz de lidar com processos estáveis, integradores e instáveis de qualquer ordem. Com base na estrutura do FSP, Torrico et al. (2013) propôs o Preditor de Smith Filtrado Simplificado (SFSP, do inglês Simplified Filtered Smith Predictor), onde o controlador primário e o filtro de referência são reduzidos a simples ganhos. No entanto, este DTC pode ser utilizado apenas para modelos de primeira ordem com atraso. Para resolver esta limitação, foi proposto o Compensador de Atraso de Transporte Simplificado (SDTC, do inglês Simplified Dead-Time Compensator) (Torrico et al., 2016), possuindo uma estrutura equivalente ao SFSP para modelos de primeira ordem, no entanto, podendo ser utilizado para modelos de ordem superior.

Apesar das vantagens do uso do SP em sistemas com atraso, problemas quanto à sua robustez foram observados em um trabalho recente de Grimholt and Skogestad (2018). Os autores observaram, nos casos estudados, uma diminuição abrupta da margem de atraso do SP, significando menor robustez à incertezas no atraso de transporte do processo para determinados níveis de robustez. 
Desse modo, o objetivo deste trabalho é mostrar a importância de estruturas derivadas do SP, avaliando a robustez à incertezas no atraso de transporte por meio de curvas de incerteza multiplicativa e de índice de robustez, um método mais conservador com relação à margem de atraso. Utilizando apenas regras de sintonia, o SDTC será comparado aos controladores $\mathrm{PI} / \mathrm{PID}$, evidenciando vantagens e desvantagens quanto ao uso desses controladores.

O texto está organizado da seguinte forma: na Seção 2, a estrutura do SDTC no domínio do tempo contínuo será descrita, na Seção 3 serão definidos os controladores PI e PID considerados, na Seção 4 serão definidos os índices de desempenho e de robustez utilizados na comparação dos controladores, na Seção 5 serão avaliados os resultados da comparação entre os controladores e, por fim, as conclusões são apresentadas na Seção 6.

\section{COMPENSADOR DE ATRASO DE TRANSPORTE SIMPLIFICADO (SDTC)}

Serão considerados neste trabalho somente modelos de processos estáveis de primeira ordem com atraso de transporte (FOPDT, do inglês first-order plus dead-time), da forma:

$$
P_{n}(s)=G_{n}(s) e^{-\theta_{m} s}=\frac{k_{m} e^{-\theta_{m} s}}{\tau_{m} s+1},
$$

onde $k_{m}$ é o ganho estático, $\tau_{m}$ é a constante de tempo, $\theta_{m}$ é o atraso de transporte e $G_{n}(s)$ é o modelo do processo livre de atraso. Para comparação entre os controladores quatro tipos de modelos serão considerados:

$$
\begin{array}{ll}
\text { Atraso de transporte puro: } & \tau_{m}=0 ; \\
\text { Dinâmica equilibrada: } & \tau_{m}=\theta ; \\
\text { Dinâmica lenta: } & \tau_{m} \geq 8 \theta ; \\
\text { Próximo de integrador: } & \tau_{m} \geq 20 \theta .
\end{array}
$$

O esquema de controle do SDTC para modelos de primeira ordem e no domínio do tempo contínuo pode ser visto na Figura 1 , onde $k_{r}$ é o filtro de referência, $k_{c}$ é o controlador primário e $V(s)$ é o filtro de robustez.

Considerando a inexistência de incertezas, quando o modelo $P_{n}(s)$ representa fielmente o processo $P(s)$, as funções de transferência para as respostas de seguimento de referência e de rejeição de perturbação são dadas, respectivamente, por:

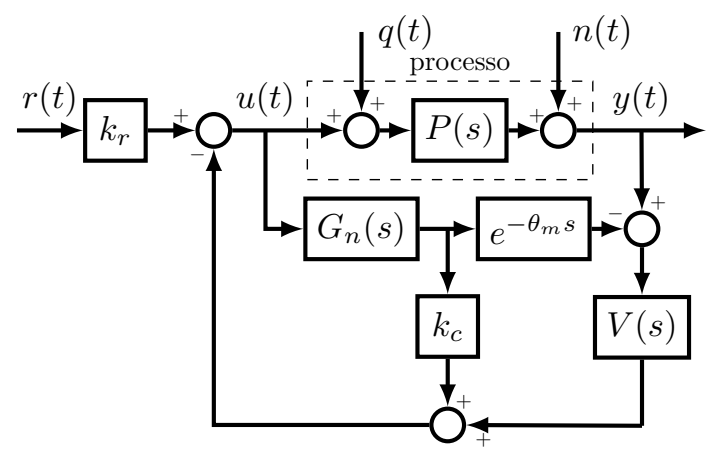

Figura 1. Esquema de controle do SDTC no domínio do tempo contínuo para modelos de primeira ordem.

$$
\begin{gathered}
H_{y r}(s)=\frac{k_{r} P_{n}(s)}{k_{c} G_{n}(s)+1}, \\
H_{y q}(s)=P_{n}(s)\left[1-\frac{P_{n}(s) V(s)}{k_{c} G_{n}(s)+1}\right] .
\end{gathered}
$$

O seguimento de referência é definido pelos ganhos $k_{r}$ e $k_{c}$, enquanto a rejeição de perturbações é definida pelo filtro $V(s)$. A seguir, os procedimentos de sintonia destes elementos para modelos FOPDT serão detalhados considerando apenas dois parâmetros livres de sintonia, constituindo os dois graus de liberdade do SDTC.

\subsection{Sintonia de $k_{r}$ e $k_{c}$}

Considerando que a resposta de malha fechada desejada para seguimento de referência deva ser a mais rápida possível e não deve apresentar sobressinal, a função de transferência de malha fechada desejada deve ser da forma

$$
H_{y r}(s)=\frac{e^{-\theta_{m} s}}{\tau_{c} s+1}
$$

onde $\tau_{c}$ é a constante de tempo de malha fechada, um dos dois parâmetros livres de sintonia do SDTC.

O filtro de referência $k_{r}$ e o controlador primário $k_{c}$ são simples ganhos, obtidos igualando as expressões (2) e (4). As expressões resultantes dependem apenas de $\tau_{c}, k_{m}$ e $\tau_{m}$ :

$$
\begin{gathered}
k_{r}=\frac{\tau_{m}}{k_{m} \tau_{c}}, \\
k_{c}=\frac{1}{k_{m}}\left(\frac{\tau_{m}}{\tau_{c}}-1\right),
\end{gathered}
$$

onde $\tau_{c} \neq \tau_{m}$.

\subsection{Sintonia do Filtro de Robustez $V(s)$}

O filtro $V(s)$ para modelos de primeira ordem é dado por

$$
V(s)=\frac{b_{1} s+b_{2}}{(\alpha s+1)^{2}}
$$

Os coeficientes $b_{1}$ e $b_{2}$ são calculados para cancelar os polos indesejados da planta na resposta de rejeição de perturbação, enquanto a constante de tempo $\alpha$ é o segundo parâmetro livre de sintonia do SDTC. Na escolha do valor de $\alpha$ existe um compromisso entre desempenho e robustez na rejeição de perturbação, de modo que quanto maior seu valor maior a robustez e quanto menor seu valor maior o desempenho.

Os coeficientes $b_{1}$ e $b_{2}$ são obtidos, respectivamente, fazendo

$$
\begin{gathered}
\left.H_{y q}(s)\right|_{s=-1 / \tau_{m}}=0 \\
\left.H_{y q}(s)\right|_{s=0}=0
\end{gathered}
$$

resultando nas expressões:

$$
\begin{gathered}
b_{1}=b_{2} \tau_{m}-\frac{k_{c}\left(\tau_{m}-\alpha\right)^{2}}{\tau_{m} e^{\frac{\theta_{m}}{\tau_{m}}}} \\
b_{2}=k_{r}
\end{gathered}
$$


Com o objetivo de alcançar maior desempenho na rejeição de perturbação, uma versão alternativa do filtro de robustez é utilizada neste trabalho. Sua expressão é dada por

$$
V(s)=\frac{b_{1} s+b_{2}}{\alpha s+1} .
$$

Os coeficientes $b_{1}$ e $b_{2}$ para esta versão do filtro de robustez são obtidos a partir de (8) e (9) como

$$
\begin{gathered}
b_{1}=b_{2} \tau_{m}+\frac{k_{c}\left(\alpha-\tau_{m}\right)}{e^{\frac{\theta_{m}}{\tau_{m}}}}, \\
b_{2}=k_{r} .
\end{gathered}
$$

Considerando que a versão alternativa do filtro $V(s)$ é de primeira ordem, essa versão do SDTC será chamada de SDTC-FO (do inglês, first-order).

\subsection{Regras de Sintonia do SDTC}

As regras de sintonia do SDTC para sistemas estáveis são resultado de um trabalho ainda não publicado. $\mathrm{O}$ valor de $\tau_{c}=0.5 \cdot \tau_{m}$ foi definido com o objetivo de se obter um bom compromisso entre velocidade de resposta e robustez. A regra $\alpha=0.539 \cdot \theta_{m}$ foi obtida por meio da aproximação linear de resultados de sintonia por otimização quando define-se a sensibilidade de pico do sistema em malha fechada em $M_{S}=1.59$.

\section{CONTROLADORES PI E PID}

O controlador PI utilizado está na forma ideal,

$$
C_{P I}(s)=K_{c}\left(1+\frac{1}{\tau_{I} s}\right)
$$

enquanto que o controlador PID está na estrutura em cascata,

$$
C_{P I D}(s)=K_{c}\left(\frac{\tau_{I} s+1}{\tau_{I} s}\right)\left(\frac{\tau_{D} s+1}{\tau_{F} s+1}\right) .
$$

Usualmente, a constante de tempo $\tau_{F}$ do filtro derivativo é um valor muito pequeno, como $\tau_{F}=\tau_{D} / 10$.

\subsection{Regras de sintonia dos controladores PI e PID}

A sintonia do controlador PI utiliza as regras de Controle de Modelo Interno Simples (SIMC, do inglês Simple Internal Model Control), propostas em Skogestad (2003), com um grau de liberdade, definido pela constante de tempo de malha fechada $\tau_{c}$ da resposta de seguimento de referência, descrita como em (4). Estas regras de sintonia para modelos FOPDT são mostradas na Tabela 1. Para os controladores PID, as regras SIMC foram melhoradas em Grimholt and Skogestad (2013), onde o termo derivativo $\tau_{D}=\theta_{m} / 3$ é definindo para esses tipos de modelo. Além disso, em modelos com atraso de transporte puro o controlador PI é somente um integrador, na forma

onde $k_{i}$ é sintonizado como

$$
C_{P I}(s)=k_{i} / s,
$$

$$
k_{i}=\frac{1}{k_{m}} \frac{\tau_{m}}{\left(\tau_{c}+\theta_{m}\right)} .
$$

\section{4. ÍNDICES DE DESEMPENHO E ROBUSTEZ}

Para a análise de desempenho, é utilizado o custo ponderado $J(p)$, definido em Grimholt and Skogestad (2012). Este índice é uma função dos parâmetros $p$ dos controladores e dos índices IAE para perturbações do tipo degrau na entrada $\left(I A E_{d q}\right)$ e na saída $\left(I A E_{d n}\right)$ do processo $P(s)$. Sua expressão é dada por

$$
J(p)=\frac{1}{2}\left(\frac{I A E_{d q}(p)}{I A E_{q}^{o}}+\frac{I A E_{d n}(p)}{I A E_{n}^{o}}\right) .
$$

Os índices de desempenho $I A E_{q}^{o}$ e $I A E_{n}^{o}$ são os mesmos utilizados em Grimholt and Skogestad (2018). Visando uma comparação consistente, esses fatores de ponderação são utilizados no cálculo da função custo tanto para o SDTC quanto para os controladores PI e PID. Nota-se que um maior desempenho é quantificado pelo menor valor de $J$, ao passo que uma menor capacidade de rejeição de perturbações está associada a um maior valor de $J$.

As funções sensibilidade $S(s)$ e sensibilidade complementar $T(s)$ são utilizadas para avaliar a robustez dos controladores, sendo definidas como

$$
S(s)=\frac{1}{C(s) G(s)+1},
$$

$$
T(s)=\frac{C(s) G(s)}{C(s) G(s)+1}=1-S(s) .
$$

Os valores máximos destas funções são obtidos por análise no domínio da frequência, onde

$$
\begin{aligned}
& M_{S}=\max _{\omega}\left|\frac{1}{C(j \omega) G(j \omega)+1}\right|, \\
& M_{T}=\max _{\omega}\left|\frac{C(j \omega) G(j \omega)}{C(j \omega) G(j \omega)+1}\right| .
\end{aligned}
$$

Como em Grimholt and Skogestad (2018), para análise de robustez, escolhe-se o maior valor entre $M_{S}$ e $M_{T}$ (Garpinger and Hägglund, 2008), sendo definido o índice $M_{S T}$ :

$$
M_{S T}=\max \left\{M_{S}, M_{T}\right\}
$$

A análise de robustez considera também a estabilidade do sistema devido a incertezas no atraso de transporte. O valor da margem de atraso $\left(M_{a}\right)$ (dada em segundos) define a máxima incerteza no atraso $\theta$ do processo antes do sistema de malha fechada se tornar instável (Åström et al., 2006). Seu valor é calculado por

Tabela 1. Regras de sintonia para os controladores PI/PID.

\begin{tabular}{ccc} 
& PI & PID \\
\hline$K_{c}$ & $\frac{1}{k_{m}} \frac{\tau_{m}}{\left(\tau_{c}+\theta_{m}\right)}$ & $\frac{1}{k_{m}} \frac{\tau_{m}}{\left(\tau_{c}+\theta_{m}\right)}$ \\
$\tau_{I}$ & $\min \left\{\tau_{m}, 4\left(\tau_{c}+\theta_{m}\right)\right\}$ & $\min \left\{\tau_{m}, 4\left(\tau_{c}+\theta_{m}\right)\right\}$ \\
$\tau_{D}$ & - & $\theta_{m} / 3$ \\
\hline
\end{tabular}




$$
M_{a}=\frac{\pi}{180^{\circ}} \cdot \frac{M_{f}}{\omega_{c}},
$$

onde $\omega_{c}$ é a frequência de cruzamento $(\mathrm{em} \mathrm{rad} / \mathrm{s})$ e $M_{f}$ é a margem de fase do sistema (em graus).

Para um sistema contendo múltiplas frequências de cruzamento $\omega_{c_{i}}$, a margem de atraso é calculada por

$$
M_{a}=\frac{\pi}{180^{\circ}} \cdot \min \left(\frac{M_{f_{i}}}{\omega_{c_{i}}}\right) .
$$

Outra forma de quantificar a robustez à incertezas no atraso de trasporte é a partir das curvas de incerteza multiplicativa $\delta P(s)$ e de índice de robustez $I_{r}$. A mínima distância entre estas curvas pode avaliar, de forma mais conservadora que a margem de atraso, a robustez do sistema em malha fechada. Seja o processo $P(s)$ escrito na forma:

$$
P(s)=P_{n}(s)[1+\delta P(s)]
$$

a incerteza multiplicativa $\delta P(s)$ é calculada como:

$$
\delta P(s)=\frac{P(s)}{P_{n}(s)}-1 .
$$

Ao considerar incertezas somente no atraso de transporte, tem-se que:

$$
\delta P(s)=e^{-\Delta \theta s}-1,
$$

onde $\Delta \theta=\theta-\theta_{m}$ representa a incerteza no atraso de transporte.

No diagrama de Nyquist, a distancia entre o ganho de malha do sistema e o ponto crítico $s=-1+j 0$ deve ser superior às incertezas no processo. O controlador $C(s)$ afeta a parcela de $P(s)$ definida como $P_{n}(s) \delta P(s)$, referente às incertezas multiplicativas. Assim, para que a condição de estabilidade seja satisfeita, tem-se que:

$$
\left|1+C(s) P_{n}(s)\right|>\left|C(s) P_{n}(s) \delta P(s)\right|,
$$

ou seja, a máxima incerteza multiplicativa deve satisfazer

$$
|\delta P(s)|<\frac{\left|1+C(s) P_{n}(s)\right|}{\left|C(s) P_{n}(s)\right|},
$$

onde o lado direito da equação (31) é definido como o índice de robustez $I_{r}$. Portanto, para que um sistema em malha fechada atenda às condições de estabilidade é necessário que

$$
I_{r}>|\delta P(s)|
$$

para valores de $s=j \omega$, onde $0 \leq \omega<\infty$.

Portanto, respeitando-se a condição (32), tem-se garantia de estabilidade em malha fechada. No entanto, caso a condição seja violada, o que ocorre quando as duas curvas de robustez se tocam, o sistema em malha fechada ainda pode ser estável. Daí a característica conservadora do índice $I_{r}$ para avaliar robustez à incertezas no atraso de transporte.

Quando em uma dada frequência $\omega$ o índice de robustez é igual à incerteza multiplicativa, o valor da incerteza é definido como máxima incerteza robusta no atraso de transporte $\Delta \theta_{\max }$, o qual limita a condição de estabilidade. De modo geral $\Delta \theta_{\max }<M_{a}$, sendo, desse modo, uma medida mais conservadora do que a margem de atraso. Portanto, ao garantir a condição descrita em (32) o sistema em malha fechada é estável e a robustez em termos da margem de atraso é também satisfeita.

\section{RESULTADOS COMPARATIVOS}

\subsection{Desempenho e Robustez à Incertezas no Atraso de Transporte}

O desempenho dos controladores quantificado pelo valor de $J$ é mostrado na Figura 2 para o valores de robustez $1 \leq M_{S T} \leq 2$. De modo geral, para os diferentes tipos de modelo FOPDT, o desempenho do SDTC é melhor para sintonias mais agressivas, onde no caso de atraso puro, por exemplo, observa-se uma diferença significativa entre o seu desempenho e o do controlador PI. Para cada tipo de modelo, pode-se comparar o SDTC ao controlador PI e o SDTC-FO ao controlador PID, visto que o desempenho destes é similar de maneira geral.

$\mathrm{Na}$ Figura 3, tem-se os valores de margem de atraso para o mesmo intervalo de $M_{S T}$. Para os quatro tipos de modelo, o SDTC e o SDTC-FO apresentam uma diminuição significativa na margem de atraso para valores de $M_{S T} \geq 1.8$. Essa característica de grande variação na margem de atraso foi também notada para o preditor de Smith em Grimholt and Skogestad (2018).

Pode-se observar que o uso do SDTC para valores de $M_{S T}$ acima de 1.8 apresenta menor robustez à incertezas no atraso quando quantificada pela margem de atraso. Entretanto, como mostra a Figura 4, ao ser analisada a máxima incerteza robusta no atraso de transporte $\Delta \theta_{\max }$ para o SDTC, não há variação brusca desta medida de robustez para diferentes valores de $M_{S T}$. Portanto, fica clara a influência de sua característica conservadora na avaliação da robustez quanto à incertezas no atraso de transporte.

\subsection{Análise no Domínio do Tempo}

Nesta seção o desempenho e a robustez dos controladores PI e SDTC são avaliadas por meio de resposta de seguimento de referência e de rejeição de perturbação utilizando o processo com dinâmica equilibrada e a sintonia para $M_{S T}=2.0$.

Na Figura 5 são apresentadas as respostas dos dois controladores para o caso nominal. Tanto na rejeição de perturbação quanto no seguimento de referência, o SDTC possui uma resposta mais rápida, confirmando o que também pode ser observado na Figura 2.

A Figura 6 apresenta as respostas dos dois controladores para uma máxima incerteza robusta no atraso $\Delta \theta_{\max }=$ +0.452 . Observa-se que as respostas de ambos controladores são estáveis e que o SDTC ainda é aquele com resposta mais rápida e que menos é influenciado pela incerteza.

\subsection{Comentários}

Na industria, o uso de curvas de incerteza multiplicativa e de índice de robustez no projeto de DTCs é uma prática já consolidada. Além disso, por causa de sua característica conservadora, utilizando o conceito de máxima incerteza 

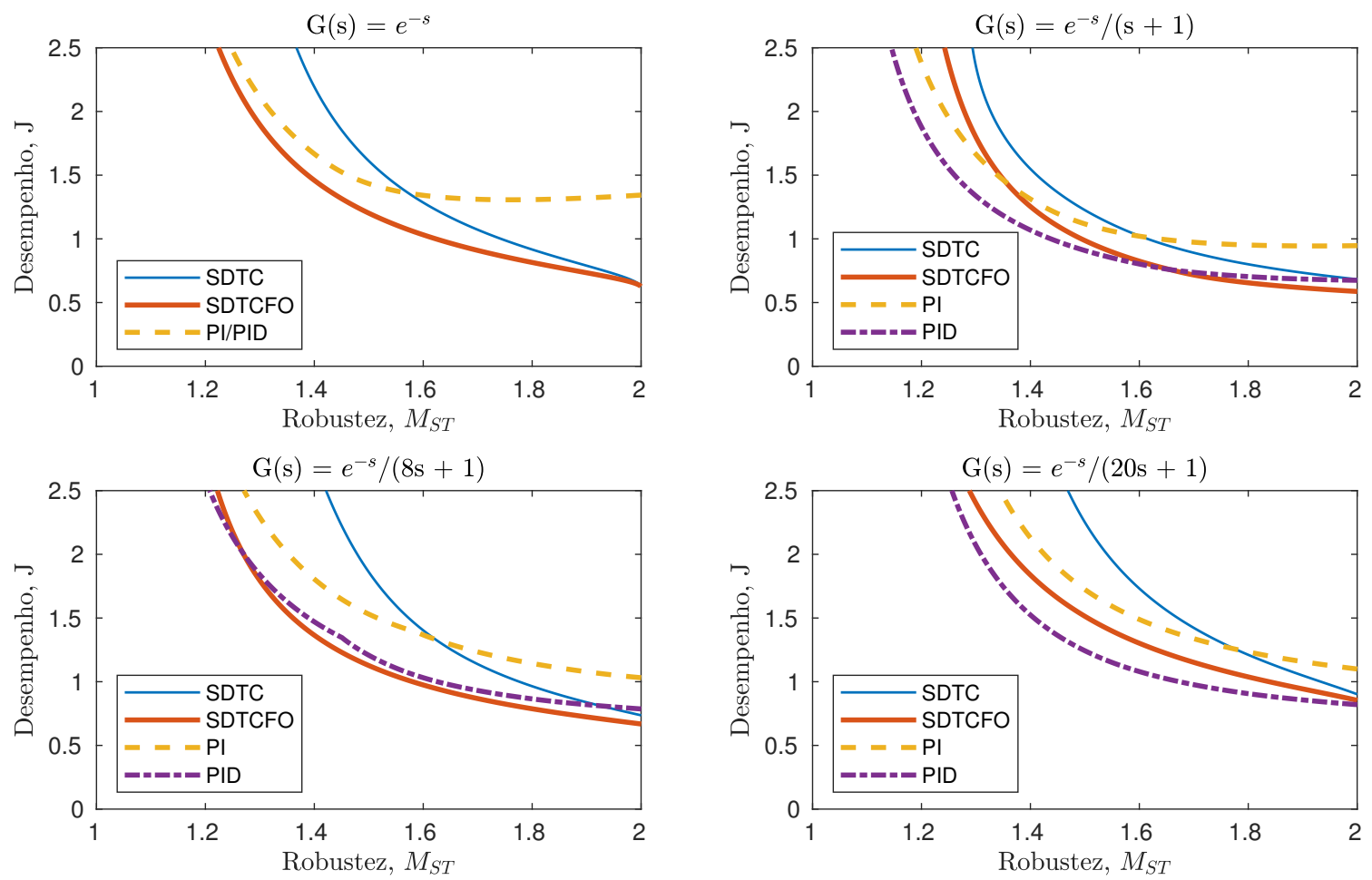

Figura 2. Índice de desempenho $J$ em função do índice de robustez $M_{S T}$ para quatro tipos de modelo FOPDT.
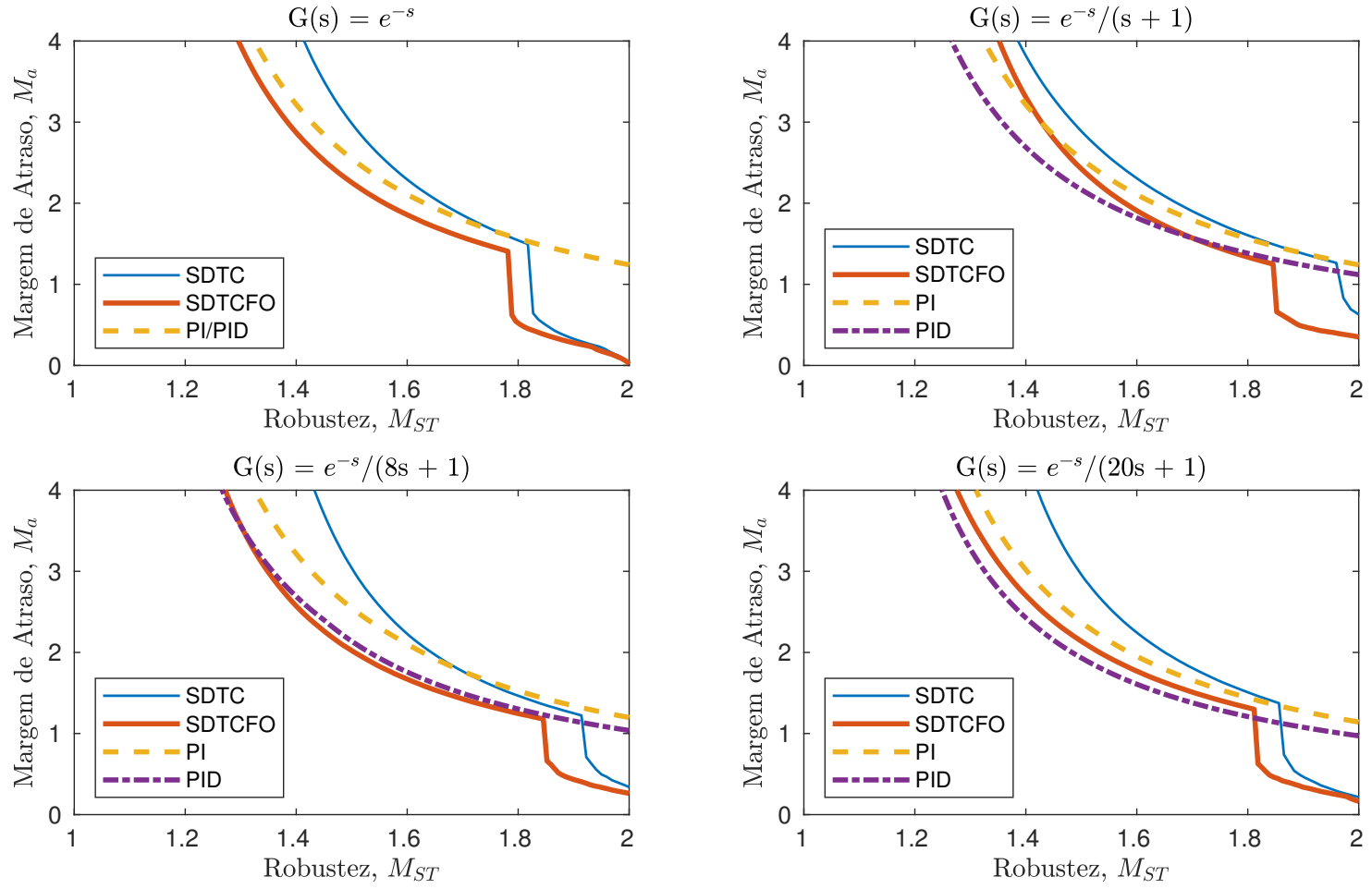

Figura 3. Margem de atraso $M_{a}$ em função do índice de robustez $M_{S T}$ para quatro tipos de modelo FOPDT.

robusta no atraso baseado em curvas de robustez, garantese também a robustez do sistema em termos de margem de atraso. Mesmo que o sistema resulte estável com a máxima incerteza robusta no atraso, é provável que, na prática, as oscilações devido à incerteza já comprometam a operação do sistema de controle. 


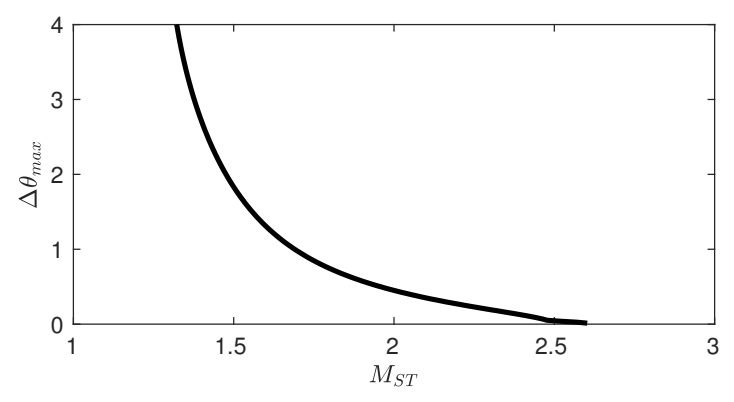

Figura 4. Máxima incerteza robusta no atraso de transporte $\Delta \theta_{\max }$ em função do índice de robustez $M_{S T}$ do SDTC para o modelo de dinâmica equilibrada.

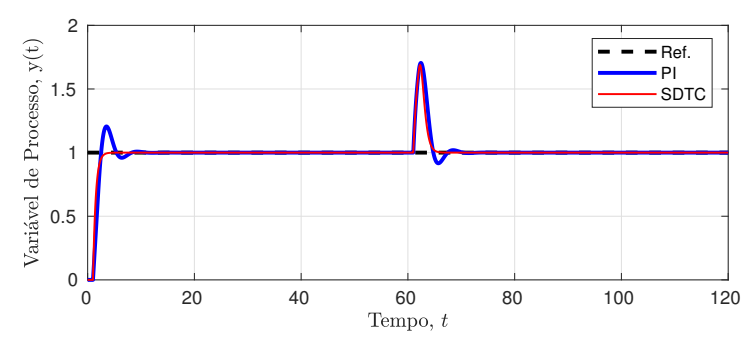

Figura 5. Variável de processo $y(t)$ para um processo com dinâmica equilibrada $G(s)=e^{-s} /(s+1)$, sem incerteza no atraso de transporte, fixado $M_{S T}=2.0$.

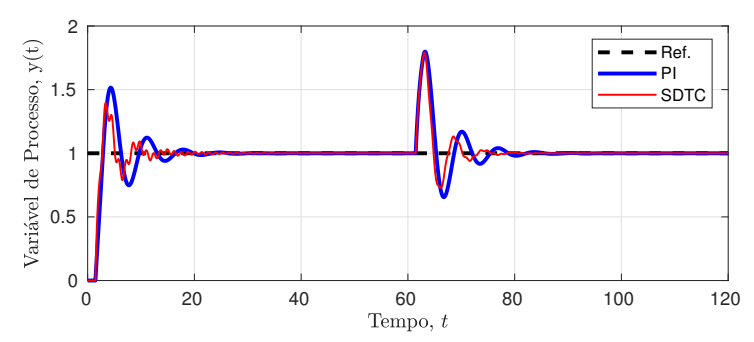

Figura 6. Variável de processo $y(t)$ para um processo com dinâmica equilibrada $G(s)=e^{-s} /(s+1)$, com incerteza $\Delta \theta=+0.452$ no atraso de transporte, fixado $M_{S T}=2.0$.

Por apresentar melhor desempenho, para sintonias muito agressivas o uso do SDTC e do SDTC-FO é mais vantajoso em relação aos controladores PI e PID em situações em que se tem um bom modelo. Já quando existem incertezas elevadas na modelagem do processo, controladores PI e PID bem sintonizados propiciam melhor desempenho.

\section{CONCLUSÃO}

Os resultados mostrados neste trabalho evidenciam que a utilização de estruturas derivadas do Preditor de Smith ainda é bastante vantajosa, visto que, a robustez à incertezas no atraso de transporte pode ser quantificada também por curvas de robustez, um método mais conservador, já bastante difundido na industria e que garante também a robustez quanto à margem de atraso.

Dado o bom desempenho do SDTC em relação a controladores PI e PID, sua utilização em aplicações que necessitam de sintonias muito agressivas continua a ser recomendada quando se possui um modelo consistente.
Atualmente, ferramentas avançadas de identificação são muito acessíveis e na maioria dos casos bons modelos podem ser obtidos. Caso isto não seja possível, para este caso particular de aplicações, controladores PI e PID passam a ser a melhor opção.

Como trabalhos futuros, será estudada a sintonia por otimização do SDTC para processos estáveis, integradores e instáveis e será feita a comparação com controladores PI/PID em termos de desempenho e robustez por meio da máxima incerteza robusta no atraso baseada em curvas de robustez, onde o objetivo de interesse é a rejeição de perturbações.

\section{AGRADECIMENTOS}

Os autores agradecem à Coordenação de Aperfeiçoamento de Pessoal de Nível Superior (CAPES) e à Coordenadoria de Acompanhamento Discente (CAD-UFC) pelo financiamento desta pesquisa.

\section{REFERÊNCIAS}

Åström, K.J., Hägglund, T., and Astrom, K.J. (2006). Advanced PID control, volume 461. ISA-The Instrumentation, Systems, and Automation Society Research Triangle.

Garpinger, O. and Hägglund, T. (2008). A software tool for robust pid design. IFAC Proceedings Volumes, 41(2), 6416 - 6421. 17th IFAC World Congress.

Grimholt, C. and Skogestad, S. (2012). Optimal pi-control and verification of the simc tuning rule. IFAC Proceedings Volumes, 45(3), 11 - 22. 2nd IFAC Conference on Advances in PID Control.

Grimholt, C. and Skogestad, S. (2013). Optimal pidcontrol on first order plus time delay systems \& verification of the simc rules. IFAC Proceedings Volumes, 46(32), 265-270.

Grimholt, C. and Skogestad, S. (2018). Should we forget the smith predictor? IFAC-PapersOnLine, 51(4), 769 774. 3rd IFAC Conference on Advances in ProportionalIntegral-Derivative Control PID 2018.

Normey-Rico, J.E. and Camacho, E.F. (2009). Unified approach for robust dead-time compensator design. Journal of Process Control, 19(1), 38 - 47.

Normey-Rico, J.E. and Camacho, E.F. (2007). Control of Dead-Time Processes. Springer.

Skogestad, S. (2003). Simple analytic rules for model reduction and pid controller tuning. Journal of Process Control, 13(4), $291-309$.

Smith, O.J. (1957). Closed control of loop with dead time. Chemical engineering progress, 53, 217-219.

Torrico, B.C., Cavalcante, M.U., Braga, A.P.S., NormeyRico, J., and Albuquerque, A.A.M. (2013). Simple tuning rules for dead-time compensation of stable, integrative, and unstable first-order dead-time processes. Industrial 85 Engineering Chemistry Research, 52, 11646-11654.

Torrico, B.C., Correia, W.B., and Nogueira, F.G. (2016). Simplified dead-time compensator for multiple delay siso systems. ISA Transactions, 60, $254-261$.

Visioli, A. and Zhong, Q.C. (2011). Control of Integral Processes With Dead-Time. Springer. 